\title{
Stewart-Treves Syndrome: A Case Report and Review of the Literature
}

\author{
Roberto Berebichez-Fridman ${ }^{\mathrm{a}, \mathrm{b}} \quad$ Yehuda E. Deutsch $^{\mathrm{c}} \quad$ Thomas M. Joyal $^{\mathrm{d}}$ \\ Pablo Montero Olvera ${ }^{b}$ Pasquale W. Benedetto ${ }^{e, f}$ \\ Andrew E. Rosenberg ${ }^{d}$ Daniel H. Kett ${ }^{\mathrm{g}}$
}

${ }^{a}$ The William J. Harrington Medical Training Programs for Latin America and the Caribbean, University of Miami, Miller School of Medicine, Miami, Fla., USA; ${ }^{b}$ School of Medicine, Faculty of Health Sciences, Anáhuac University, Mexico City, Mexico; Departments of ${ }^{\mathrm{C}} \mathrm{Hematology/Oncology}$ and ${ }^{\mathrm{d}}$ Pathology and Laboratory Medicine,

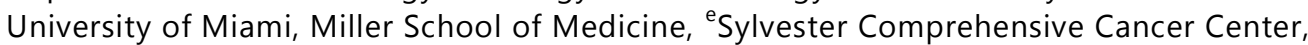
University of Miami, Miller School of Medicine, 'Department of Hematology/Oncology, Jackson Memorial Hospital, and ${ }^{9}$ Department of Critical Care Medicine, University of Miami, Miller School of Medicine, Miami, Fla., USA

\section{Key Words}

Stewart-Treves syndrome - Angiosarcoma - Lymphedema - Morbid obesity · Cancer . Diagnosis · Treatment

\begin{abstract}
The Stewart-Treves syndrome is a rare and deadly entity, which is defined as angiosarcoma arising in the setting of chronic lymphedema. It typically presents in women who develop lymphedema in the upper extremity secondary to axillary lymph node dissection for breast cancer surgery. It is extremely uncommon in the lower extremities as a result of idiopathic chronic lymphedema. Here, we present the case of a 63-year-old female patient with idiopathic chronic lymphedema of the lower extremities having morbid obesity (BMI 82.6) and multiple comorbidities. She developed multiple confluent, hemorrhagic and necrotic elevated purple-black papules in the lower extremities, for which the initial diagnosis was cellulitis. Because there was no improvement with antibiotics, a lower extremity ultrasound and biopsy was performed which showed multiple masses in the left inner upper calf with solid and cystic components. The pathology results of the punch biopsies were consistent with angiosarcoma. Immunohistochemical studies revealed positivity for CD31, FLI-1, and a high Ki-67 proliferation rate. Because of the patient's weight and medical comorbidities, no further extensive diagnostic tests were performed to detect metastatic disease, and because of contraindications, no further medical treatment was provided. The patient subsequently died 1 month after diagnosis.

(C) 2016 The Author(s)

\section{KARGER}




\section{Case Reports in Oncology}

Berebichez-Fridman et al.: Stewart-Treves Syndrome: A Case Report and Review of the Literature

\section{Case Presentation}

A 63-year-old white female patient with morbid obesity (body mass index of 82.6), chronic bilateral lower extremity lymphedema, chronic kidney disease, congestive heart failure, hypertension, type 2 diabetes, hypothyroidism, glaucoma, gastroesophageal reflux disease, chronic obstructive pulmonary disease, asthma, obesity hypoventilation syndrome and depression, presented to the hospital with shortness of breath and fatigue. She had been living in a nursing home and was completely immobile for the last 9 years secondary to both morbid obesity and lymphedema. The patient did not have a history of lower extremity surgery, trauma, or infection.

The patient developed multiple hemorrhagic and elevated purple-black lesions (fig. 1ac). The initial diagnosis by the Internal Medicine and Dermatology Department was cellulitis of the lower extremities. However, there was no clinical improvement with antibiotics. A lower extremity ultrasound was performed which showed multiple masses in the left inner upper calf, with solid and cystic components, subcutaneous edema, and necrosis. Two 3-mm punch biopsies were performed.

The pathology of the punch biopsies revealed angiosarcoma, present at deep and peripheral tissue edges. There were prominent epithelioid cytology and characteristic hemorrhage (fig. 2a, b). Immunohistochemical studies revealed that the tumor cells were positive for CD31, FLI-1 with a high Ki-67 proliferation rate (up to 30\% of total tumor cells) (fig. 2c). Melan A and pancytokeratin showed no immunoreactivity. Based on the morphology, immunoprofile, and clinical presentation, the patient was diagnosed with Stewart-Treves syndrome (STS).

Due to the patient's weight and size, additional staging workup, including computerized axial tomography, PET-CT, or magnetic resonance imaging, was unable to be performed. Based on the patient's poor baseline performance status and history of cardiac and renal disease, she was not eligible for the standard cardiotoxic and nephrotoxic chemotherapeutic agents required for treatment. Approximately 1 month after the biopsy results were obtained, the patient developed septic shock and multiorgan failure that resulted in her death.

\section{Discussion}

STS is a deadly and rare disease. It is defined as the association between chronic lymphedema and angiosarcoma [1, 2]. It was originally described in women who developed lymphedema as a result of axillary lymph node dissection associated with mastectomy for breast cancer. The presence of this syndrome significantly worsens the outcome of patients with breast cancer [2,3]. STS accounts for approximately 5\% of angiosarcomas [4].

Loewenstein in 1906 described angiosarcoma in the extremity of a patient affected by severe posttraumatic lymphedema for 5 years [3]. However, the first description in the English literature is attributed to Kettle in 1918, who described angiosarcoma in the leg of a 44year-old patient with congenital lymphedema. In 1948, Dr. Fred W. Stewart, Professor of Pathology, and Dr. Norman Treves, Associate Professor of Surgery, both from the Cornell University Medical College, New York, N.Y., USA, reported six cases of angiosarcoma arising from sites of postmastectomy lymphedema [1]. They called it 'elephantiasis chirurgica'. Prior to this report, such cases went unreported since the clinical findings were erroneously attributed to diffuse and recurrent inoperable cutaneous manifestations of breast cancer [1].

While most commonly associated with breast cancer, this syndrome can be broadly applied to lymphangiosarcoma, which develops from chronic lymphatic obstruction, either 
Berebichez-Fridman et al:: Stewart-Treves Syndrome: A Case Report and Review of the Literature

congenital or acquired. Initially, electron microscopic and immunohistological studies revealed the origin of this cancer to be from blood vessel rather than lymphatic endothelium, thereby classifying the tumor as a hemangiosarcoma [2]. However, more recent immunohistochemical studies confirmed the original description, confirming the tumor as a lymphangiosarcoma $[3,5]$.

There are approximately 400 cases of STS reported in the literature, most in postmastectomy patients who are predominantly women. The incidence of this disease is very low (between 0.07 and $0.45 \%$ of patients survive at least 5 years postradical mastectomy) [3]. Lymphedema affects approximately $14 \%$ of patients who undergo complete axillary lymph node dissection [6]. Most of these breast cancer patients had undergone a Halsted radical mastectomy [6]. There are also case reports of patients who developed STS on closed shunts in renal transplant and chronic kidney disease [7]. No racial preponderance has been observed in this syndrome. It has not been shown to be more prevalent in patients with AIDS or any other immunodeficiency disorders, as compared with Kaposi's sarcoma (KS). The peak age of presentation of angiosarcoma is between 65 and 70 years, with an average patient age of 68.8 years at diagnosis for breast cancer-associated patients. The incidence of STS has notably declined secondary to the improvement of operative techniques in breast cancer as well as radiation therapy techniques and the development of novel chemotherapy agents. In addition, the incidence of chronic lymphedema after breast cancer treatments has been reduced from 40 to $4 \%[3,8]$. Among patients receiving radiation therapy, the relative risk of developing angiosarcoma is $15.9 \%$ [3].

Patients usually have a significant history for breast cancer and radical mastectomy, usually 5-15 years before sarcoma presentation. The mean onset between radical mastectomy and lymphangiosarcoma is 11 years [3].

There are many causes of lymphedema, such as trauma, infectious (filariasis), idiopathic acquired lymphoma, venous stasis, morbid obesity, leg ulcerations, lymphoma, and surgery of the groin, legs, or pelvis. One type of lymphedema, called massive localized lymphedema, is associated with obesity, and its incidence is rising secondary to the epidemic of obesity in the Western society. Only nine cases of angiosarcoma arising in the setting of massive localized lymphedema have been reported $[6,9,10]$. These are acquired forms of lymphedema, but there can also be congenital lymphedema. Notably, chronic lymphedema caused by cardiac, hepatic, or renal disease is not associated with STS. The reason for this observation is not known. The mechanism by which some forms of chronic lymphedema can induce angiosarcoma is unknown and controversial. One theory is that chronic lymphedema causes local immunodeficiency, therefore permitting oncogenesis $[2,3,6]$. Buildup of protein-rich interstitial fluid alters the local immune response within the affected limb, stimulating lymphangiogenesis and the development of collateral vessels [11]. In addition, the fluids in the lymphatic channels are rich in growth factors [6]. Supporting the immunodeficiency hypothesis is the observation that, in 1960, a homograft skin transplant on a lymphedematous arm survived much longer than homograft skin transplanted to healthy arms. There is no evidence for a benign precursor of the angiosarcoma seen in STS [3]. Furthermore, there have been case reports of patients who developed this syndrome while taking chronic systemic immunosuppressive medications, chemotherapy, and in patients with acquired immunodeficiency syndrome. This raises the possibility that systemic, not only local immunosuppression, may have a role in the development of lymphangiosarcoma [6, 7]. Interestingly, some authors postulate that mutations in the DNA repair genes BRCA1 and BRCA2 predispose to angiosarcomas after treatment for breast cancer [2].

Three phases in the development of STS have been described: (1) prolonged lymphedema, (2) angiomatosis, and subsequently (3) angiosarcoma [12]. Prolonged 
Berebichez-Fridman et al.: Stewart-Treves Syndrome: A Case Report and Review of the Literature

lymphedema (lymphostasis) is observed in KS as well as angiosarcoma, resulting in localized immunosuppression and vascular oncogenesis in the presence of human herpesvirus 8 (HHV-8) infections and immunosuppression [3]. Lymphangiosarcoma and KS have been reported to occur together in a patient receiving immunosuppressive treatment for a kidney transplant [6]. Additionally, lymphedema has also been described in other cutaneous malignancies including melanoma, basal cell carcinoma, squamous cell carcinoma, Merkel cell carcinoma, and cutaneous lymphomas $[6,11]$.

The histologic findings of angiosarcoma in STS show a proliferation of irregular anastomosing vascular channels that dissect dermal collagen. The malignant endothelial cells lining these channels are hyperchromatic and pleomorphic with macronucleoli and frequent mitosis. The tumor cells are round to ovoid with a predominantly epithelioid cytomorphology and often project into the lumen of the vessels creating a 'hobnailed' appearance on higher magnification [8, 13]. The overlying epidermis may be acanthotic, hyperkeratotic, or atrophic, with or without proliferation of reticular fibers in the dermis [3, 14]. Interestingly, the angiosarcomas in STS present heterogeneous morphology with areas dominated by hemangiosarcoma and lymphangiosarcoma structures [8].

Histopathological and immunohistochemical confirmation is essential for diagnosis. Immunohistochemical antibodies against endothelial cells are typically the stain of choice. Not all malignant endothelial cells will be positive for this marker; thus, a panel of immunohistochemical stains should be used to avoid false-negatives. Diagnostically helpful stains include CD31, CD34, FLI-1, von Willebrand factor, and factor VIII [2, 3, 5, 7]. In addition, numerous lymphatic associated antibodies have been developed, including podoplanin (D240), lymphatic vessel endothelial hyaluronan receptor-1 (LYVE-1), prospero homeobox protein 1, and vascular endothelial growth factor receptor 3 (VEGFR-3) [2, 3, 6]. Thanks to these endothelial lymphatic markers, a possible relationship between KS and STS was made because the markers were present in both types of sarcomas. Nevertheless, examination of STS specimens with electronic microscopy further supports lymphatic origin by identifying a complete basal lamina surrounding the malignant cells. Additionally, electronic microscopy has identified pinocytosis, intracellular junctions, and cytoplasmic intermediate filaments, as well as Weiber-Palade bodies, storage granules, and erythrophagocytosis. These ultrastructural features oppose findings seen in tumors of vascular origin and support the classification as a tumor of lymphatic origin $[3,11,13]$.

Lymphedematous changes are required for the development of STS. Usually, this lymphedema occurs without a history of complications, such as thrombosis or infection. Lymphangiosarcomas are much more frequent in the upper extremity, $90 \%$ of cases present like this [3]. Moreover, lymphangiosarcomas are present in the ipsilateral side in which the radical mastectomy was performed and the lymphedema developed. The lymphedema in the upper extremities gradually extends from arm to forearm and dorsal aspects of the hand and fingers [12]. Local discomfort develops when the skin distends, but this occurs usually in advanced stages of the syndrome. Recurrent erysipelas may develop in areas of longstanding chronic lymphedema. Patients may also note atrophic skin that has become pachydermatous with wrinkle lines. Telangiectasias and hyperkeratosis may also occur [4]. The initial lesion includes either a palpable subcutaneous mass or a poorly healing eschar with recurrent bleeding. In more advanced stages, multiple reddish blue macules or nodules develop and may become polypoid. There may also be small satellite areas surrounding the nodules that may become confluent. As the lesions progress, the overlying atrophic skin becomes ulcerated, leading to infection and bleeding. Necrosis of the skin can also happen [4]. If left untreated, extensive cutaneous nodules appear and metastatic disease develops. 
Berebichez-Fridman et al:: Stewart-Treves Syndrome: A Case Report and Review of the Literature

The most common site of metastasis are the lungs, followed by liver, bone, soft-tissue structures and lymph nodes via the hematogenous route $[2,3,10,15]$.

The differential diagnosis for STS includes both benign and malignant vascular diseases. Acquired angioedema, ecchymosis, pyogenic granuloma, lymphangiectasia, angioendotheliomatosis, benign lymphangioendothelioma, lymphangioma, melanoma, and metastatic carcinoma to the skin should be considered. In addition, telangiectatic metastatic breast disease to the skin should be considered $[3,15]$. The most important differential diagnosis of STS is KS. This is a very confusing disorder because it can also develop in the presence of lymphedema, and the skin lesions and histological findings can be similar. KS differs from STS because it does not require the presence of lymphedema for its development. Immunohistochemical testing for the presence of HHV-8 is the primary way to distinguish STS from KS. KS is associated with this virus, while STS is not [2]. Some authors hypothesize that there may be a virus involved with the pathogenesis of STS $[3,12]$.

In 1959, McConnell and Haslam proposed a system for the staging of postmastectomy lymphangiosarcoma, which divides this disease in 3 stages [3]. This system lacks universal application. Histological grading is not used in staging because angiosarcomas are highgrade tumors by definition. Imaging studies can be used for staging purposes, such as CT, magnetic resonance, and radiography. Magnetic resonance is an excellent study for evaluation of the local progression of the disease, but it has limited ability to delineating tumor margins. Intravenous contrast may be used to enhance the tumor. Chest radiography and CT can be used to identify pulmonary metastases and pleural effusion $[3,10]$. For the demonstration of subcutaneous spread and planning of a surgical procedure, F-fluorodeoxyglucose positron emission tomography may be used $[3,13]$. The usefulness of sentinel lymph node biopsy is unknown [2].

The treatment of STS is complex; it usually requires medical and surgical treatment. It is recommended early amputation/disarticulation or wide local excision of the affected limb in localized disease, which offers the best chance of long-term survival. Radical ablative surgery should be done early in the diagnosis. Wide local excision is not beneficial over amputation. Even when a 2-3-cm wide margin of resection is obtained at the primary surgery, a high local recurrence rate is observed. The report by Grobmyer et al. [15] identified no significant difference in the survival of patients treated with chemotherapy versus radiation therapy. Long-term survivorship after chemotherapy/radiation is reported in case reports, but the overall results are poor [1-3, 6,13-15]. Causes of long-term survivorship of STS could in fact be secondary to undiagnosed cutaneous nodular KS. Chemotherapy agents used in this disease includes 5-fluorouracil, methotrexate, bleomycin, and/or a combination of actinomycin D, vincristine, doxorubicin, cyclophosphamide, and/or dacarbazine. Even with these treatment therapies, local recurrence and metastasis is high $[2-4,11,14]$. Treatment with immunotherapy has been demonstrated to be favorable as a palliative treatment for the management of pleural effusions caused by metastatic disease [11, 14].

It is still unclear if antilymphangiogenic treatment will provide good results. It has been hypothesized that blocking both the lymphangiogenesis and hemangiogenesis pathways may inhibit rapid growth and metastatic spread, but more studies are needed $[2,8]$.

The overall prognosis of STS is poor, with a high rate of local recurrence and metastatic disease $[2,3,16]$. The course of the disease can be complicated with recurrent erysipelas and deep venous thrombosis of the affected limb. Pain and pleural effusion also complicate the course. The median survival is 2.5 years after diagnosis, with most patients dying from metastatic disease within 2 years [2-4, 6]. The long-term survivorship is rare. Untreated patients usually live for 5-8 months after diagnosis. Preventative measures should be encouraged given the disappointing treatment results. These include treatment of the chronic 
Berebichez-Fridman et al:: Stewart-Treves Syndrome: A Case Report and Review of the Literature

lymphedema, weight loss, pressure garments, physiotherapy, compressive devices, microsurgery, and laser therapy [3, 4, 6, 15].

\section{Statement of Ethics}

All reasonable efforts to obtain consent for publication from the patient's next of kin failed, as they were untraceable. The authors have made every effort to ensure patient anonymity. There is no reason to believe that the patient would have objected to publication and it is not felt that anyone who knew the patient would be able to identify her from the published article.

\section{Disclosure Statement}

The authors declare that they have no competing interests.

\section{References}

1 Stewart FW, Treves N: Lymphangiosarcoma in postmastectomy lymphedema: a report of six cases in elephantiasis chirurgica. Cancer 1948;1:64-81.

2 Young RJ, Brown JN, Reed MW, Hughes D, Woll PJ: Angiosarcoma. Lancet Oncol 2010;11:983-991.

3 Sharma A, Schwartz RA: Stewart-Treves syndrome: pathogenesis and management. J Am Acad Dermatol 2012;67:1342-1348.

4 McHaffe DR, Kozak KR, Warner TF, Cho CS, Heiner JP, Attia S: Stewart-Treves syndrome of the lower extremity. J Clin Oncol 2010;28:351-352.

5 Wysochi WM, Komorowski A: Stewart-Treves syndrome. J Am Coll Surg 2007;205:194-195.

-6 Tabareau F, de Muret A, Miquelestorena E, Decouvelaere AV, de Pinieux G: Cutaneous epithelioid clear cells angiosarcoma in a aoung woman with congenital lymphedema. Case Rep Pathol 2013;1:1-6.

7 Kamijo F, Kiniwa Y, Goto Y, Shirota S, Kobayashi A, Tanaka A, Fukuzawa M, Saida T: A case of angiosarcoma arising on a closed shunt in a patient with chronic renal failure. J Am Acad Dermatol 2011;65:882-883.

-8 Lee R, Saardi KM, Schwartz RA: Lymphedema-related angiogenic tumors and other malignancies. Clin Dermatol 2014;32:616-620.

-9 Stanczyk M, Gewartowska M, Swierkowski M, Grala B, Maruszynski M: Stewart-Treves syndrome angiosarcoma expresses phenotypes of both blood and lymphatic capillaries. Chin Med J 2013;126:231-237.

$\$ 10$ Yamane H, Ochi N, Tabayashi T, Takigawa N: Stewart-Treves syndrome after cervical cancer. Int Med 2012;51:513.

11 Shon W, Ida CM, Boland-Froemming JM, Rose PS, Folpe A: Cutaneous angiosarcoma arising in massive localized lymphedema of the morbidly obese: a report of five cases and review of the literature. J Cutan Pathol 2011;38:560-564.

12 Aygit AC, Yildirim AM, Dervisoglu S: Lymphangiosarcoma in chronic lymphoedema. J Hand Surg 1999;24B:135-137.

13 di Meo N, Drabeni M, Gatti A, Trevisan G: Letter: A Stewart-Treves syndrome of the lower limb. Dermatol Online J 2014;18:1-2.

14 Ho J, Jin Y, Young D, Wha S, Won J, Tae S: Clinical Experience of Stewart- Treves Syndrome in the Lower Leg. Arch Plast Surg 2013;40:275-277.

15 Grobmyer S, Daly J, Glotzbach R, Grobmyer A: Role of surgery in the management of postmastectomy extremity angiosarcoma (Stewart-Treves syndrome). J Surg Oncol 2000;73:182-188.

16 Taswell HF, Soule EH, Coventry MB: Lymphangiosarcoma arising in chronic lymphedematous extremities. J Bone Joint Surg 1962;44:277-294. 


\section{Case Reports in Oncology}

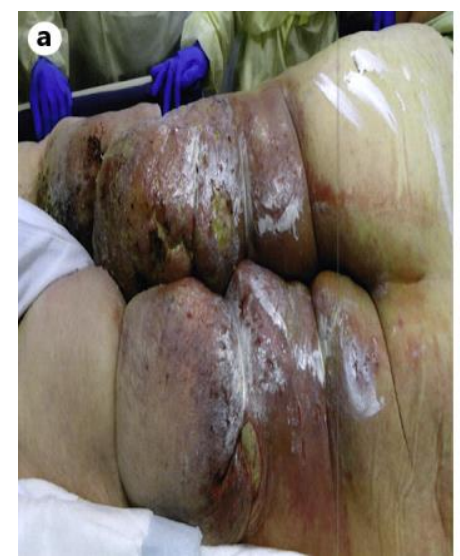

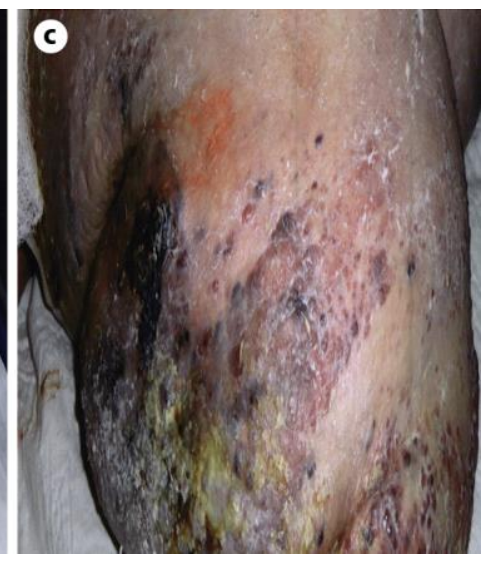

Fig. 1. a Lower extremities of the patient. The morbid obesity and the lymphedema are evident. Also noted are some pressure ulcers in the posterior aspect of the legs with signs of infection. $\mathbf{b}$ Multiple hemorrhagic and elevated purple-black lesions as well as multiple small satellite lesions in the lower extremity. c Close-up of the multiple purple-black lesions of the lower extremity, with hemorrhagic characteristics and signs of infection.
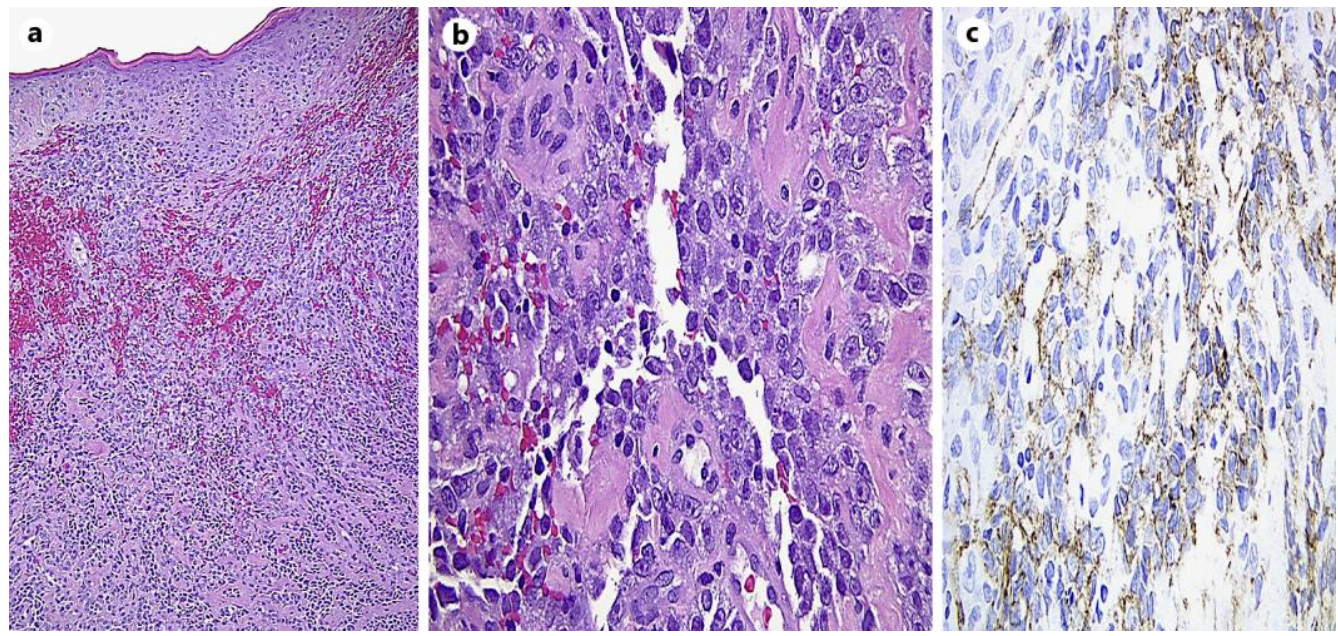

Fig. 2. a Hematoxylin and eosin. $\times 100$. The neoplasm infiltrates through the dermis and is associated with hemorrhage. b Hematoxylin and eosin. $\times 400$. Irregular vascular spaces are lined by layers of cytologically malignant epithelioid endothelial cells that have amphophilic cytoplasm, large vesicular nuclei with prominent nucleoli. c CD31. $\times 400$. Immunohistochemical stain for CD31 stains neoplastic endothelial cells in a membranous pattern. 